\title{
SchoOl ScREenING FOR ScOLIOSIS
}

ABSTRACT: School screening for scoliosis can be highly effective in detecting those relevant curvatures that would benefit most from the available conservative treatment at an advantageously early stage. The correct age group of

\section{CONNELLAN K'}

'University of the Witwatersrand adolescent females is targeted, and routine examination can be administered with relative ease.

The program is most accurate when performed with a primary Forward Bending Test, and in cases where scoliosis is suspected, a second screening using either the Forward Bending examination or a scoliometer reading is recommended before referral to a co-operating medical team. It is convenient, and suitably accurate following brief training, for the first two tests to be conducted by the school's nurse or physical education staff

Screening at schools allows for early diagnosis of scoliosis, allowing maximum benefit and prognosis to be obtained as a result of the subsequent non-operative treatment. Boston braces have presented the highest rates of compliance and have thus yielded some of the most beneficial results of management by bracing. A treatment is deemed successful if the progression of the curvature has been stopped, and this occurs with the majority of cases that are detected whilst they are in the early stages. The prognosis of scoliosis is therefore improved by conservative treatment measures.

The cost of implementing a school screening program is justified by its ability to decrease the morbidity of those adolescents who are affected by this disease. The programs are generally inexpensive, are not time consuming and are simple to administer. As much as $75 \%$ to $95 \%$ of AIS cases that require surgery could have avoided operative management through early diagnosis and treatment. As the screening projects encourage and implement the early detection of scoliosis, which can then be effectively managed, there is great value in the implementation and maintenance of these school screening programs. 